\title{
EFFECT OF THE INCLINE ANGLE OF PROPELLER BOSS CAP FINS (PBCF) ON SHIP PROPELLER PERFORMANCE
}

\author{
Insanu Abdilla Cendikia Abar ${ }^{1 *}$, I Ketut Aria Pria Utama ${ }^{1}$ \\ ${ }^{1}$ Institut Teknologi Sepuluh Nopember, Jl. Raya ITS, Keputih, Kec. Sukolilo, Kota Surabaya 60111, \\ Indonesia
}

(Received: August 2018 / Revised: December 2018 / Accepted: July 2019)

\begin{abstract}
In order to understand the effect of modifying the traditional form of propeller hub into the propeller boss cap fins (PBCF) form, a series of tests was conducted to discover the best type. Analysis was made using the computational fluid dynamic (CFD) approach, together with ANSYS CFX code. Two types of hub were employed, namely convergent and divergent. Both types were made using slope angles of 5, 10 and 15 degrees. Comparative analysis of the data was made, combined with validation by published papers. The overall results indicate that compared to a normal hub, the traditional convergent type has an increased efficiency of around $1.4 \%$, while the divergent type decreases efficiency by approximately $1.2 \%$. Furthermore, the PBCF convergent hub results in increased efficiency of around $0.8 \%$, whereas the divergent type decreases efficiency by about $1.0 \%$. This study is in good agreement with previous papers, with a discrepancy of approximately $2 \%$.
\end{abstract}

Keywords: Energy saving devices; Hub vortex; Propeller boss cap fins

\section{INTRODUCTION}

Propeller boss cap fins (PBCF) have been used since 1988 as an innovative energy saving device in marine transportation, according to the International Towing Tank Conference (ITTC). The addition of PBCF can improve the efficiency of a ship's propeller. The other function of PBCF is to eliminate the vortex phenomenon on the hub part of the rotor. It has been evidenced by Dang et al. (2011), Kawamura et al. (2012), Cheng and Hao-Eng (2014), Molland et al. (2014), and Sun et al. (2016) in research based on field studies, lab trials and computational fluid dynamic (CFD) simulations that PBCF can eliminate the vortex and improve the efficiency of ships' propellers. Considering design parameters, $\mathrm{PBCF}$ will influence propeller efficiency noticeably (Seo et al., 2016; Mizzi et al., 2017).

In the past few decades, research on PBCF geometry has been conducted to discover which components of PBCF are highly sensitive to efficiency and the hub vortex phenomenon. Several papers (Ghassemi et al., 2012; Druckenbrod et al., 2015; Kimura et al., 2018) have found that variations in PBCF fin position are highly influential on propeller efficiency and added hub configurations indicate can affecting vortex in the hub cap area. This has led to the indication that the shape of the hub geometry has an effect on the shape and magnitude of the vortex, as stated by Katayama et al. (2015). The geometry of the hub propeller is divided into three parts, convergent, straight and divergent, with each type having a different vortex characteristic.

\footnotetext{
${ }^{*}$ Corresponding author's email: insanu.abar.93@gmail.com, Tel. +62-858-6727-2792 Permalink/DOI: https://dx.doi.org/10.14716/ijtech.v10i5.2256
} 
In addition, Katayama et al. (2015) undertook research on the addition of updated PBCF using convergent hub types, and obtained good efficiency. However, this does not apply to the research on the type of divergent hub conducted by Lim et al. (2014), who found decreased propeller system efficiency. These two issues are the basis for this research.

CFD simulation focuses on varying the inclined angle of the hub cap (convergent and divergent types) and then converting it into PBCF. Furthermore, each type of hub cap has varying incline angles of 5, 10 and 15 degrees. Comparison is made between each type of conventional and PBCF hub in order to obtain the best results.

\section{METHODOLOGY}

In the early stages, the propeller data were modelled by CAD software and analyzed by CFD; those used in this simulation were taken from Prakash and Nath (2012). The main size of the propeller can be seen in Table 1.

Table 1 Main propeller dimensions

\begin{tabular}{lc}
\hline \multicolumn{1}{c}{ Parameter } & Dimension \\
\hline Diameter & $2 \mathrm{~m}$ \\
Pitch Ratio & 0.8 \\
Expanded Area & 0.55 \\
Blade Number & 4 \\
$\mathrm{RPM}$ & 257 \\
$\mathrm{Va}$ & $4.87 \mathrm{~m} / \mathrm{s}$ \\
\hline
\end{tabular}

\subsection{Meshing, Boundary Condition and Validation}

The investigation was conducted using ANSYS CFX code, with the application of an unstructured grid or mesh. The boundary conditions were adjusted according to the experiment performed by Chamanara and Ghasemmi (2016), as shown in Figures 1a and 1b. The testing was conducted under turbulent flow conditions, with the use of k-epsilon turbulence modelling (Davidson, 2018).

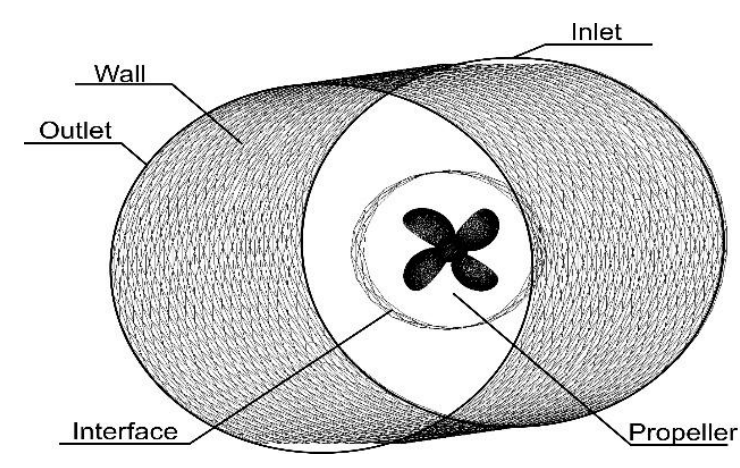

(a)

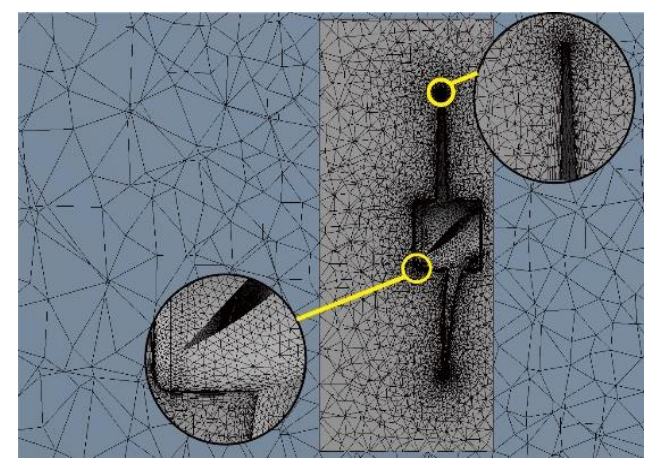

(b)

Figure 1 (a) Boundary conditions; (b) Meshing

A grid independence is the addition of the number of an element to obtain a constant value,so that the numerical result close to the experiment result., Comparison of errors between numerical and experiment cannot be more than 2\% (Almohammadi et al., 2013; Suastika et al., 2017). Table 2 shows a summary of the thrust coefficient from different numbers of elements. It was discovered that by using 1,041,759 elements the error was around $1.8 \%$ and the simulation time 2 hours 45 minutes. Table 2 shows the mean error percentage between the previous and new grid. 
Table 2 Grid independence

\begin{tabular}{lccccc}
\hline Number of Elements & 250,176 & 501,085 & $1,041,759$ & $2,107,532$ & $4,192,311$ \\
\hline $\mathrm{K}_{\mathrm{T}}$ & 0.116 & 0.132 & 0.143 & 0.146 & 0.149 \\
Percentage & - & $8.4 \%$ & $6.2 \%$ & $1.8 \%$ & $1.5 \%$ \\
Time Simulation & $35 \mathrm{~m}$ & $1 \mathrm{~h} 15 \mathrm{~m}$ & $2 \mathrm{~h} 45 \mathrm{~m}$ & $6 \mathrm{~h} 23 \mathrm{~m}$ & $12 \mathrm{~h} 10 \mathrm{~m}$ \\
\hline
\end{tabular}

Analysis was performed using a standard k-epsilon turbulence model with a second order (high resolution) order of accuracy in the calculations. The results for the propeller model without hub cap variation were compared with previous analysis (Prakash \& Nath, 2012), as shown in Table 3. The gap between the previous results and the present CFD appears to be satisfactory, with a $\mathrm{K}_{\mathrm{T}}$ value gap between the previous and present results of $1.4 \%$, and a $\mathrm{K}_{\mathrm{Q}}$ gap percentage of around $7 \%$. The difference between the two analyses is below $10 \%$, so according to CFD standard scan be considered appropriate.

Table 2 Validation model

\begin{tabular}{lccc}
\hline & $\mathrm{K}_{\mathrm{T}}$ & $10 \mathrm{~K}_{\mathrm{Q}}$ & $\eta_{0}$ \\
\hline Prakash and Nath (2012) & 0.142 & 0.201 & 0.623 \\
Present CFD & 0.144 & 0.217 & 0.599 \\
\hline
\end{tabular}

The simulation for the present CFD can be seen in Figure 2. It has been adjusted to the previous analysis in order to compare the results and validity of the CFD model. It appears that there is a small vortex slightly to the right side and in front of the propeller hub, therefore hub modification is needed; the addition of PBCF can reduce or even remove the vortex phenomenon. The results from the simulation with regard to the thrust coefficient $\left(\mathrm{K}_{\mathrm{T}}\right)$, torque coefficient $\left(\mathrm{K}_{\mathrm{Q}}\right)$ and Open

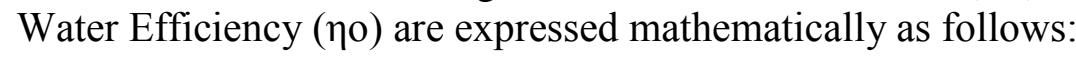

$$
\begin{gathered}
K_{T}=\frac{T}{\rho n^{2} D^{4}} \\
K_{Q}=\frac{Q}{\rho n^{2} D^{5}} \\
\eta \mathrm{O}=\frac{V a}{2 \pi n D} \frac{K_{T}}{K_{Q}}
\end{gathered}
$$

where $\rho$ is the density of water, $n$ is revolutions per second of the propeller, $D$ is the diameter of the propeller and $\mathrm{Va}$ is the velocity of advance.
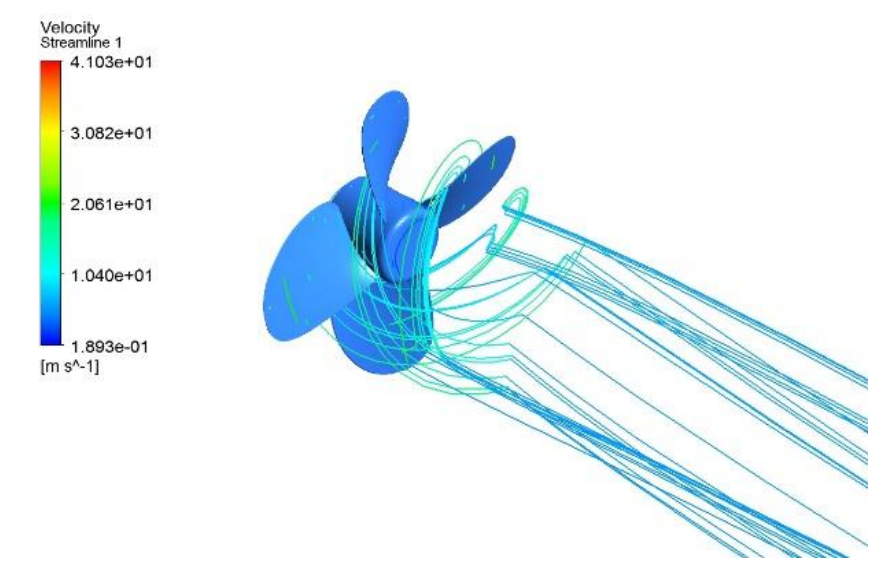

Figure 2 CFD simulation without modification 
After validating the 3D model, variations to the propeller rpm were made, with the results shown in Table 4. These indicate that optimum thrust, torque and efficiency were obtained at $256 \mathrm{rpm}$.

Table 4 Validation model

\begin{tabular}{cccc}
\hline Rpm $(\mathrm{n})$ & $\mathrm{K}_{\mathrm{T}}$ & $10 \mathrm{~K}_{\mathrm{Q}}$ & Efficiency \\
\hline 1461 & 1.341 & 3.333 & 0.364 \\
974 & 0.704 & 1.333 & 0.503 \\
256 & 0.144 & 0.217 & 0.601 \\
209 & 0.055 & 0.102 & 0.488 \\
146 & 0.003 & 0.016 & 0.202 \\
\hline
\end{tabular}

\section{RESULTS AND DISCUSSION}

\subsection{Calculation of the Performance of a Conventional Variation Cap}

Analysis focused on the inclined angle from two types of hub cap propeller. The shape of the variation can be seen in Figure 4.

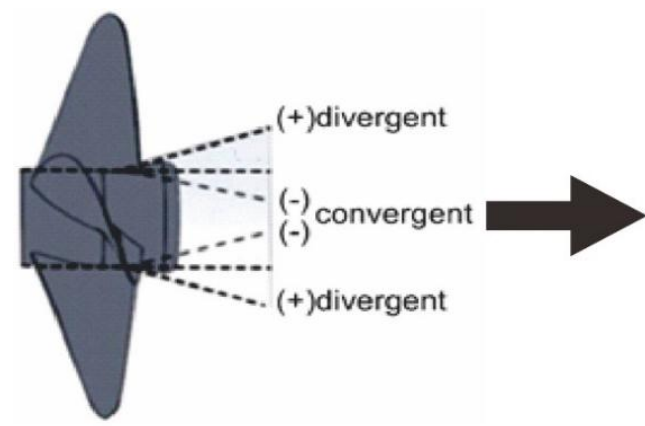

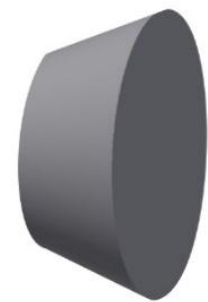

(a) divergent

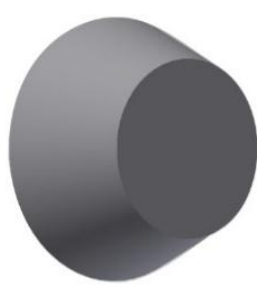

(b) convergent

Figure 3 Inclined angle variation

The study selected two types of hub cap, namely convergent and divergent, according to Katayama et al. (2015); the form of both hub caps can be seen in Figure 3. Figure 3a shows the divergent type with conical geometry, whilst Figure $3 b$ shows a convergent type hub cap, with the shape of the geometry extending outwards. Both hub cap types have varying inclined angles of 5, 10 and 15 degrees. The results are shown in Tables 5 and 6.

Table 5 Results of (-) convergent cap variation

\begin{tabular}{lcccc}
\hline \multirow{2}{*}{ Parameter } & \multicolumn{4}{c}{ Inclined Angle } \\
\cline { 2 - 5 } & $0^{\circ}$ & $5^{\circ}$ & $10^{\circ}$ & $15^{\circ}$ \\
\hline $\mathrm{K}_{\mathrm{T}}$ & 0.143 & 0.144 & 0.144 & 0.145 \\
$10 \mathrm{~K}_{\mathrm{Q}}$ & 0.217 & 0.212 & 0.209 & 0.209 \\
Efficiency & 0.599 & 0.614 & 0.624 & 0.625 \\
\hline
\end{tabular}

Table 6 Results of (+) divergent cap variation

\begin{tabular}{lcccc}
\hline \multirow{2}{*}{ Parameter } & \multicolumn{4}{c}{ Inclined Angle } \\
\cline { 2 - 5 } & $0^{\circ}$ & $5^{\circ}$ & $10^{\circ}$ & $15^{\circ}$ \\
\hline $\mathrm{K}_{\mathrm{T}}$ & 0.143 & 0.139 & 0.138 & 0.138 \\
$10 \mathrm{~K}_{\mathrm{Q}}$ & 0.217 & 0.212 & 0.214 & 0.214 \\
Efficiency & 0.599 & 0.599 & 0.584 & 0.583 \\
\hline
\end{tabular}


It can be seen from Table 4 that there is an increase in the $\mathrm{K}_{\mathrm{T}}$ value of $0.2 \%$, whilst $\mathrm{K}_{\mathrm{Q}}$ decreases at a rate of $1.4 \%$. Table 5 shows that the value of $\mathrm{K}_{\mathrm{T}}$ decreases by around $1 \%$, while $\mathrm{K}_{\mathrm{Q}}$ increases by $1 \%$. It can be concluded from Figure 4 that the convergent cap produces a positive result, with an increase in $\mathrm{K}_{\mathrm{T}}$, a decrease in $\mathrm{K}_{\mathrm{Q}}$ and an increase in efficiency at each degree of variation.

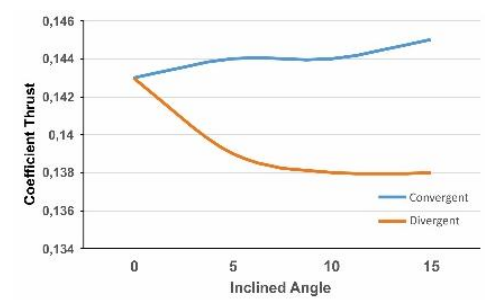

(a) $\mathrm{K}_{\mathrm{T}}$ vs Slope angle

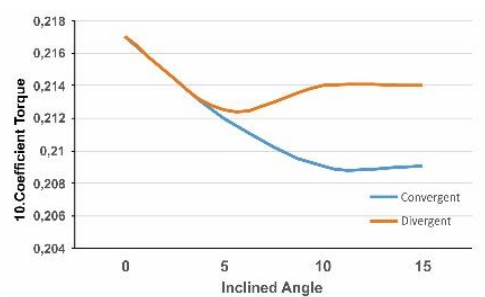

(b) $\mathrm{K}_{\mathrm{Q}}$ vs Slope angle

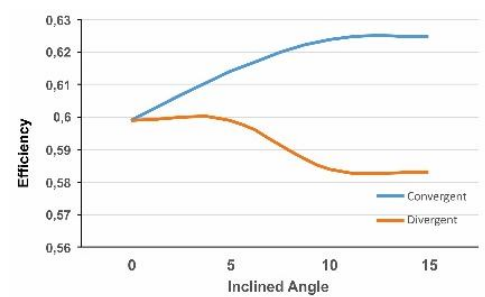

(c) Efficiency vs Slope angle

Figure 4 Graphic Comparison Conventional Hub

The phenomenon of the increment of $\mathrm{K}_{\mathrm{T}}$ and decrement of $\mathrm{K}_{\mathrm{Q}}$ is, according to Islam et al. (2006) mathematically influenced by the effect of the pressure coefficient, by which the increase in pressure area will cause the pressure value to decrease. Efficiency is affected by the values of thrust and torque generated by the propeller. The calculations made from Table 4 show that the value of efficiency experienced an increase of $1.4 \%$, while those from Table 5 indicate that the value decreased by approximately $1.2 \%$.

\subsection{Calculation of Performance based on PBCF Variation}

The conventional hub cap was analyzed, followed by PBCF analysis. The conventionally-shaped hub cap modified into PBCF can be seen in Figure 5.

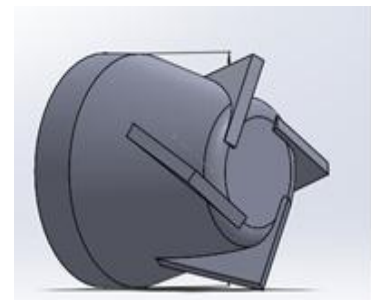

(a)

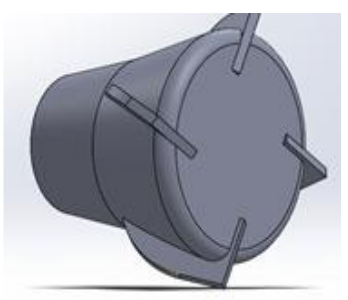

(b)

Figure 5 Design of PBCF

Figure 5a shows a convergent-type hub cap modified into a PBCF type, while Figure 5b shows a hub cap with a divergent-type modification. Both PBCF types have varying inclined angles of 5, 10 and 15 degrees. The results are shown in Tables 7 and 8.

Table 7 Results of PBCF with (-) convergent type modification

\begin{tabular}{lcccc}
\hline \multirow{2}{*}{ Parameter } & \multicolumn{4}{c}{ Inclined Angle } \\
\cline { 2 - 5 } & $0^{\circ}$ & $5^{\circ}$ & $10^{\circ}$ & $15^{\circ}$ \\
\hline $\mathrm{K}_{\mathrm{T}}$ & 0.144 & 0.146 & 0.147 & 0.148 \\
$10 \mathrm{~K}_{\mathrm{Q}}$ & 0.217 & 0.215 & 0.213 & 0.213 \\
Efficiency & 0.604 & 0.617 & 0.628 & 0.629 \\
\hline
\end{tabular}


Table 8 Results of PBCF with (+) divergent type modification

\begin{tabular}{lcccc}
\hline \multirow{2}{*}{ Parameter } & \multicolumn{4}{c}{ Inclined Angle } \\
\cline { 2 - 5 } & $0^{\circ}$ & $5^{\circ}$ & $10^{\circ}$ & $15^{\circ}$ \\
\hline $\mathrm{K}_{\mathrm{T}}$ & 0.144 & 0.144 & 0.142 & 0.141 \\
$10 \mathrm{~K}_{\mathrm{Q}}$ & 0.216 & 0.217 & 0.218 & 0.222 \\
Efficiency & 0.604 & 0.601 & 0.589 & 0.575 \\
\hline
\end{tabular}

It can be seen from Tables 7 and 8 that the results for the two types of hub after modification into PBCF demonstrate the same trend this can be seen in Figure 6, where the value of $K_{T}$ in the convergent type increases, whilst in the divergent one it decreases. A similar phenomenon occurs with the efficiency trend, but not with the $\mathrm{K}_{\mathrm{Q}}$ one.

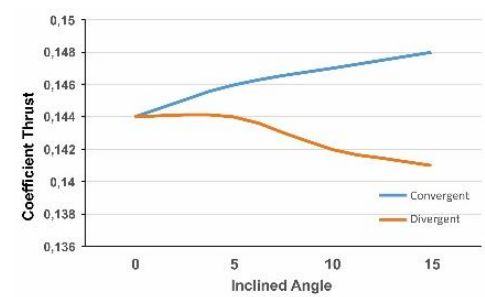

(a) $\mathrm{K}_{\mathrm{T}}$ vs Slope angle

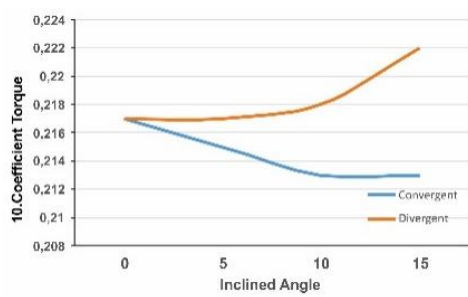

(b) $\mathrm{K}_{\mathrm{Q}}$ vs Slope angle

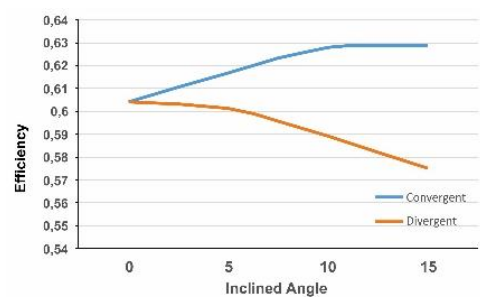

(c) Efficiency vs Slope angle

Figure 6 Graphic PBCF Comparison

Although the PBCF results show the same trend as the conventional ones, it has been found that an increase in efficiency occurred in each type of hub after modification into PBCF. In the case of the convergent cap on PBCF in Table 8 compared with the conventional cap in Table 6 , there is an increment in efficiency of up to $0.8 \%$. In addition, the divergent cap on PBCF in Table 7 also shows an increment in efficiency of up to $0.8 \%$, while at an angle of $15^{\circ}$ efficiency decreases by $1 \%$. The phenomenon of increased efficiency after modification into PBCF appears to be due to the improvement of the stress phenomena occurring in the hub cap region, as described by Ouchi et al. (1988) and Nojiri et al. (2011); with added PBCF the pressure on the cap area will be well distributed, hence the negative pressure will change and increase the pressure value. Seo et al. (2016) explain that after a test on a towing tank, it was shown that the hub vortex on divergent types caused the performance of the propeller to decline. The occurrence of pressure drops often cause the phenomenon of cavitation due to the frequent occurrence of friction fluid, which makes the flow more turbulent. Furthermore, cavitation causes a loss of thrust in marine propellers due to the reduction in the contact area between the blades and water, and thus efficiency degradation (Asimakopoulos \& Kaklis, 2016).

\subsection{Hub Vortex Simulation}

The simulation of a hub vortex was performed in two stages: before and after modification with PBCF. Figure 7 shows the simulation on a conventional hub cap.

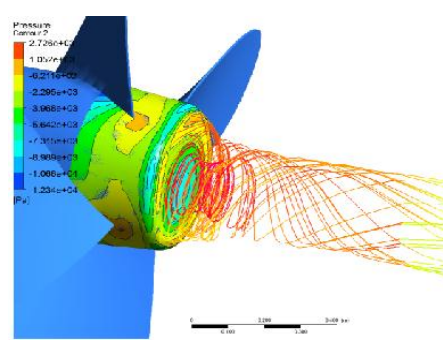

(a) Conv. cap $5^{\circ}$

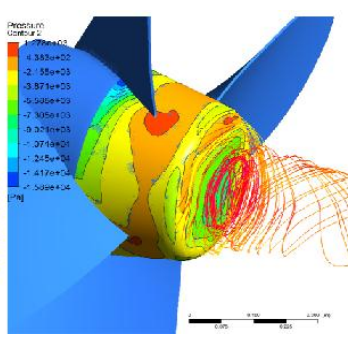

(b) Conv. cap $10^{\circ}$

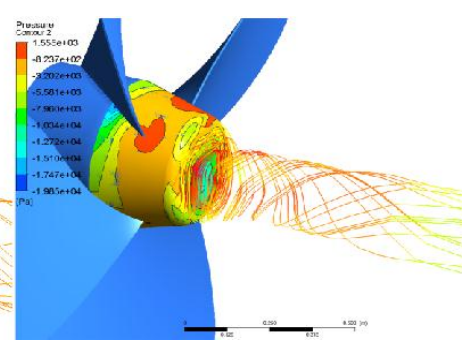

(c) Conv. Cap $15^{\circ}$ 


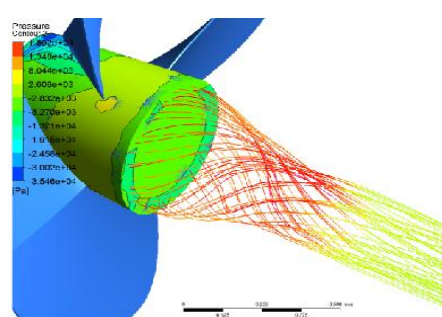

(a) Div. cap $5^{\circ}$

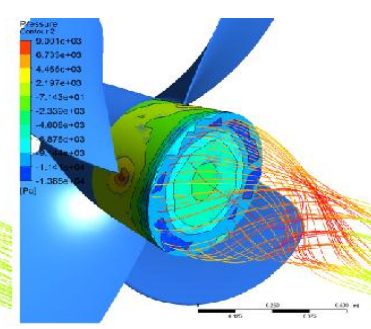

(b) Div. cap $10^{\circ}$

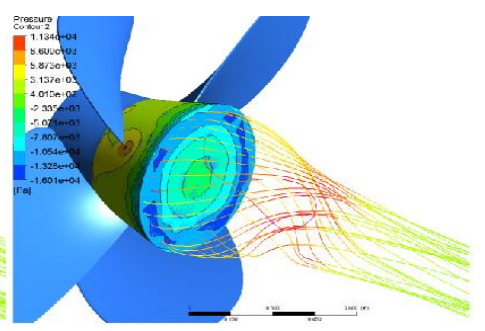

(C) Div. cap $15^{\circ}$

Figure 7 Conventional cap pressure contours and streamlines

Figures $7 \mathrm{a}-7 \mathrm{c}$ show that the pressure increase makes the negative pressure area smaller. Moreover, Figures $7 \mathrm{~d}-7 \mathrm{f}$ show that the pressure value decreases and spreads throughout the area at the back of the divergent hub cap. In the convergent case, the decrease in negative pressure causes a reduction in the area of the hub cap.

The pressure concentration on the hub affects the flow, as shown in Figure 7. The convergent cap forms a focused flow, as can be seen in Figures $7 \mathrm{a}-7 \mathrm{c}$, whereas the divergent cap forms a wide flow from the hub cap area, as shown in Figures $7 d-7 f$. The increase in the area of the hub cap will reduce the pressure that occurs around the hub cap region, hence causing the hub vortex phenomenon. According to Katayama et al. (2015), the convergent form of cap is most likely to have negative pressure at its end due to its form being conical. Meanwhile, the divergent cap with the width of the hub geometry causes uniform pressure on the cap area at the back. This negative pressure phenomenon indicates the occurrence of vortex hubs and excessive cavitation bubbles in the hub cap, as found by Ghassemi et al. (2012) and Seo et al. (2016), which are marked by a change in color in the hub cap area approaching cyan blue. Figure 8 shows the simulations on the conventional cap modified into PBCF.

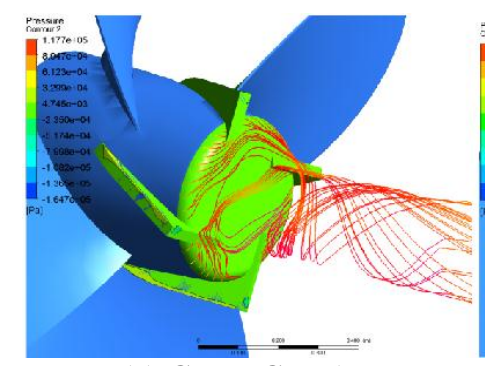

(a) Conv. Cap $5^{\circ}$

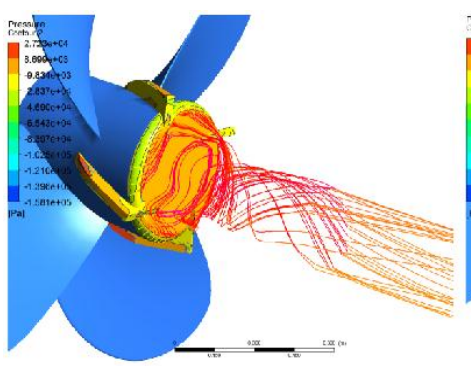

(a) Div. cap $5^{\circ}$

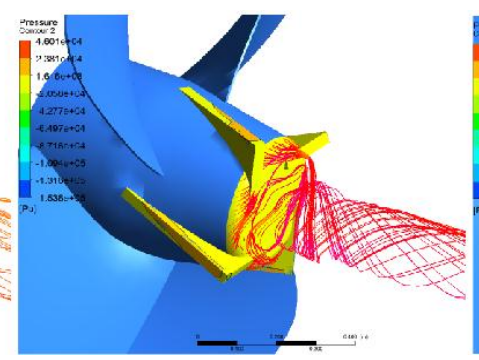

(b) Conv. Cap $10^{\circ}$

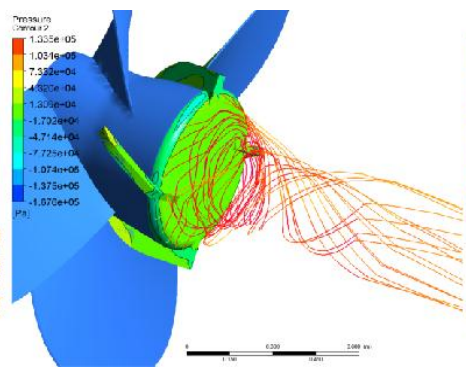

(b) Div. cap $10^{\circ}$

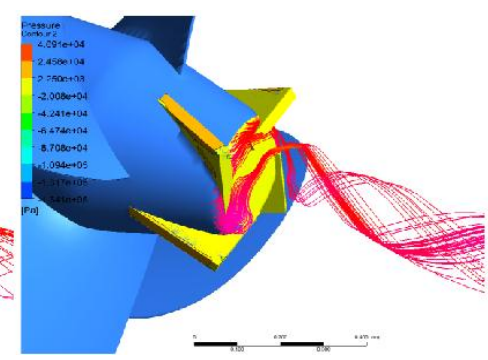

(C) Conv. Cap $15^{\circ}$

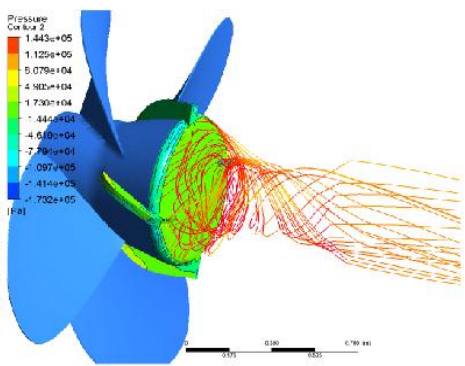

(C) Div. cap $15^{\circ}$

Figure 8 PBCF Pressure Contours and Streamlines

Modification of the conventional cap into PBCF can minimize the existence of hub vortices. This is indicated by the increase in the pressure contours around the hub cap area. It can be seen in Figures $7 \mathrm{a}$ to $7 \mathrm{f}$ that the pressure contour is more colorful around the hub cap area, being an indication of irregular pressure. Meanwhile, after conversion into PBCF (see Figures 8a to 8f), it was found that the pressure color spreads more evenly, showing regularly distributed pressure. 
The absence of fins (see Figures $7 \mathrm{a}$ to $7 \mathrm{f}$ ) shows a lower pressure contour value, which is increased by adding fins, as shown in Figures $8 \mathrm{a}$ to $8 \mathrm{f}$. This is an indication that fins can minimize negative pressure on the propeller hub and is in line with the work conducted by Ouchi et al. (1989) and Nojiri et al. (2011). Furthermore, in the convergent cap, the addition of PBCF successfully reduces the occurrence of vortices and improves the flow quality, as can be seen in Figure $8 \mathrm{a}-8 \mathrm{c}$. On the other hand, for the divergent cap, the addition of PBCF does not work optimally because it does not cover all the parts of the hub cap, as shown in Figure $8 \mathrm{~d}-8 \mathrm{f}$.

\section{CONCLUSION}

CFD has been fairly successfully used to simulate and demonstrate the use of PBCF on propeller hub caps. The results are excellent for the convergent hub, whilst the divergent one shows a disappointing output. The convergent hub increases efficiency by around $1.4 \%$ compared to conventional one, which rises further by approximately $0.8 \%$ after being converting into PBCF. On the other hand, the divergent hub decreases efficiency by around $1.2 \%$, with a further decrease of approximately $1 \%$ after being converting into PBCF.

In addition, the incline angle can influence the increase or decrease in efficiency. The reason for this is attributed to the decrease in the pressure area on the convergent hub and the increase on the divergent one. This occurs because in the case of divergent hubs the pressure drops and the shape of the flow is affected, resulting in the emergence of hub vortices.

\section{REFERENCES}

Almohammadi, K.M., Ingham, D.B., Ma, L., Pourkashan, M., 2013. Computational Fluid Dynamics (CFD) Mesh Independency Techniques for a Straight Blade Vertical Axis Wind Turbine. Journal of Energy, Volume 58, pp. 483-493

Asimakopoulos, O.A., Kaklis, P., 2016. Effect of Propeller Geometry on Cavitation. $4^{\text {th }}$ Edition. University of Strathclyde, Glasgow, Scotland: Year Individual Project

Chamanara, M., Ghasemmi, H., 2016. Hydrodinamic Characteristics of the Kort - Nozzle Propeller by Different Turbulent Models. American Journal of Mechanical Engineering, Volume 4(5), pp. 169-172

Cheng, M., Hao-Eng, C., 2014. The Design of Propeller and Propeller Boss Cap Fins (PBCF) by an Integrative Method. Journal of Hydrodinamic, Volume 26(4), pp. 586-593

Dang, J., Chen, H., Dong, G., 2011. An Exploratory Study on the Working Principle of Energy Saving Devices (ESD). Symposium on Green Ship Technology Greenship, Volume 2(1), pp. $50-59$

Davidson, L., 2018. An Introduction to Turbulence Models. Chalmers University of Technology, Goteborg, Sweden

Druckenbrod, M., Wang, K., Greitsch, L., Heinke, H.J., Abdel-Maksoud, M., 2015. Development of Hub Cap Fitted with PBCF. International Symposiums on Marine Propulsors, Volume 3(1), pp. 350-358

Ghassemi, H., Mardan, A., Ardeshir, A., 2012. Numerical Analysis of Hub Effect on Hydrodinamic Performance of Propellers with Inclusion of PBCF to Equalize the Induced Velocity. The Journal of Gdansk University of Technology, Volume 19(2), pp. 17-24

Islam, M.F., Veitch, B., Bose, N., Liu, P., 2006. Numerical Study of Effects of Hub Taper Angle on the Performance of Propellers Designed for Podded Propulsion Systems. In: The Thirteenth Annual Conference of the Computation Fluid Dynamics Society of Canada, Volume 39(2), pp. 31-37

Katayama, K., Okada, Y., Okazaki, A., 2015. Optimization of the Propeller with ECO-cap by CFD. International Symposiums on Marine Propulsors, Volume 3(1), pp. 358-365 
Kawamura, T., Ouchi, K., Takeuchi, S., 2012. Model and Full Scale CFD Analysis of Propeller Boss Cap Fins (PBCF). Journal of Marine Science and Technology, Volume 17(4), pp. 469 480

Kimura, L., Ando, S., Ono, S., Tanaka, Y., Takeuchi, S., Asanuma, N., 2018. Investigation on Full Scale Performance of the Propeller Bos Cap Fins (PBCF). International Full Scale Ship Performance, Volume 2, pp. 73-81

Lim, S.S., Kim, T.W., Lee, D.M., Kang, C.G., Kim, S.Y., 2014. Parametric Study of Propeller Boss Cap Fins for Container Ships. International Journal Naval Architecture Ocean Engineering, Volume 6(2), pp. 187-205

Mizzi, K., Demirel, Y.K., Banks, C., Turan, O., Kaklis, P., Atlar, M., 2017. Design Optimisation of Propeller Boss Cap Fins for Enhanced Propeller Performance. Journal of Ocean and Marine Engineering, Volume 62, pp. 210-222

Molland, A.F., Turnock, S.R., Hudson, D.A., Utama, I.K., 2014. Reducing Ship Emission: A Review of Potensial Practical Improvements in the Propulsive Efficiency of Future Ships. Transactions of the Royal Institution of Naval Architects Part A: International Journal of Maritime Engineering, Volume 156, pp. 175-188

Nojiri, T., Ishii, N., Kai, H., 2011. Energy Saving Technology of PBCF (Propeller Boss Cap Fins) and Its Evolution. Japan Institute of Marine Engineering, Volume 46(3), pp. 350-358

Ouchi, K., Ogura, M., Kono, Y., Orito, H., Shiotsu, T., Tamashima, M., Koizuka, H., 1988. A Research and Development of PBCF (Propeller Boss Cap Fins) - Improvement of Flow from Propeller Boss. Journal of the Society of Naval Architects of Japan, Volume 1988(163), pp. 66-78

Ouchi, K., Tamashima, M., Kawasaki, T., Koizuka, H., 1989. Research and Development of PBCF (Propeller Boss Cap Fins). Journal of the Society of Naval Architects of Japan, Volume 1989(165), pp. 43-53

Prakash, S., Nath, D.R., 2012. A Computational Method for Determination of Open Water Performance of a Marine Propeller. International Journal of Computer Applications, Volume 58(12), pp. 431-436

Seo, J., Lee, S.J., Han, B., Rhee, S.H., 2016. Influence Design Parameter Variation for PropellerBoss-Cap-Fins on Hub Vortex Reduction. Journal of Ship Research, Volume 60(4), pp. 203 218

Suastika, K., Hidayat, A., Riyadi, S., 2017. Effects of the Application of a Stern Foil on Ship Resistance: A Case Study of an Orela Crew Boat. International Journal of Technology,Volume 8(7), pp. 1266-1275

Suastika, K., Nugraha, F., Utama, I., 2017. Parallel-Middle-Body and Stern-form Relative Significance in the Wake Formation of Single-screw Large Ships. International Journal of Technology, Volume 8(1), pp. 94-103

Sun, Y., Su, Y., Wang, X., Hu, H., 2016. Experimental and Numerical Analyses of the Hydrodynamic Performance of Propeller Boss Cap Fins in a Propeller Rudder System. Engineering Applications of Computational Fluid Mechanics, Volume 10(1), pp. 145-159 